\title{
AN OVERVIEW OF RECENT ADVANCES IN SYSTEM IDENTIFICATION
}

\author{
Jer-Nan Juang * \\ NASA Langley Research Center \\ Hampton, Virginia 23681
}

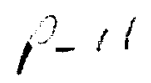

\begin{abstract}
This paper presents an overview of the recent advances in system identification for modal testing and control of large flexible structures. Several techniques are discussed including the Observer/Kalman Filter Identification, the Observer/Controller Identification and the State-Space System Identification in the Frequency Domain. The System/Observer/Controller Toolbox developed at NASA Langley Research Center is used to show the applications of these techniques to real aerospace structures such as the Hubble spacecraft telescope and the active flexible aircraft wing.
\end{abstract}

\section{INTRODUCTION}

Since the mid-sixties the field of system identification has been an important discipline with the automatic control area. ${ }^{1}$ One reason is the requirement that mathematical models within a specified accuracy must be used to apply modern control methods. Another reason is the availability of digital computers which can perform complex computations. Since then, there are a multitude of approaches, perspectives and techniques to be used for system identification. Most techniques are found very useful for application to the electrical engineeering problems. Nevertheless, most techniques do have difficulties in application to other areas such as the large aerospace structures which can only be accurately described by a large-size model with the dimension in the order of hundreds. In addition, most large aerospace structures possess significant uncertainties and nonlinearities which make system identification even more difficult, if not impossible.

In aerospace structures, there are basically three types of identification work, namely modal parameter identification, structural-model parameter identification and control-model identification. All three types of identification are important technology areas and they have different principal objectives and histories of development. The modal parameter identification and structural-model parameter identification are used in structural engineering whereas the control-model identification is used in control of flexible structures.

In this paper, we will focus on the modal parame-

\footnotetext{
*Principal Scientist, Spacecraft Dynamics Branch
}

ter identification and the control-model identification. Modal parameter identification, which is generally referred to as modal testing in the field of structures, means the process of measuring signals produced by a structure and identifying modal parameters (i.e., damping, frequencies, mode shapes and modal participation factors). System identification in the field of controls means the process of measuring signals produced by a system and building a control-model to represent the system for control design. If the identified model is a linear model in state space representation, the eigensolution of the model provides eigenvalues and eigenvectors which in turn determine modal parameters for structures. Correlation between the fields of modal testing and system identification for controls is evident.

In the past decade, many system identification techniques were developed and/or applied to identify a state space model for modal parameter identification of large flexible structures. The identified state space model is also used in controller design. Many satisfactory results were reported in the literature. ${ }^{2,3}$ Most techniques are based on sampled pulse or impulse system response histories which are known as Markov parameters. The usual practice uses the Fast Fourier Transforms (FFT) of the inputs and measured outputs to compute the sampled pulse response histories. The discrete nature of the FFT causes one to obtain pulse response rather than impulse response, and a somewhat rich input is required to prevent numerical ill-conditioning in the computation. Another approach is to solve directly in the time domain for the Markov parameters from the input and output data The drawbacks of this method include the need to invert an input matrix which necessarily becomes particularly large for lightly damped systems. ${ }^{4}$

Recently, a method has been developed to compute the Markov parameters of a linear system, which are the same as its pulse response history. ${ }^{5-12}$ The method, referred to as the Observer/Kalman Filter Identification algorithm (OKID) is formulated entirely in the time domain, and is capable of handling general response data. A fundamental difference in this approach is the introduction of an observer in the identification equations. This makes identification possi- 
ble for not only the open-loop system, but also an associated observer which can be later used in controller design. Depending on the noise characteristics, the method identifies a deadbeat observer which is the fastest possible observer in the absence of noises, or a Kalman filter which is an optimal observer in the presence of noises, or any other observer with user specified poles. The method has been successfully applied to identification of real systems, including a linear model of the space shuttle remote manipulator based on a non-linear simulation code, ${ }^{13}$ and the Hubble space telescope. ${ }^{14}$

An important extension of the above OKID method is the identification of closed-loop systems. There are several instances when such a need arises. The system may be operating in closed-loop and only closed-loop data is available for identification. An open-loop model of the system may be required to be identified from closed-loop data for the purpose of structural analysis or controller re-design. Certain systems such as an aircraft under the flutter condition are inherently unstable. For such systems, it may not be desirable or even passible to remove the existing feedback control system to perform open-loop identification. For the case where the existing controller dynamics is assumed to be unknown, a method was developed in Ref. 15, referred to as the Observer/Controller Identification algorithm (OCID), to identify an openloop model, and an effective observer/controller combination. The case where the closed-loop system does not possess a full state feedback structure, but rather a controller with known output feedback dynamics, is treated in a separate paper. ${ }^{16}$ The mathematical formulations for the two cases are entirely different since the former case deals with known feedback control signals, whereas the latter case deals with known feedback controller dynamics.

It has been found that the OKID method can effectively identify the state space models using time domain input-output data. However, there are cases in which frequency response data, rather than time histories, are available. This is often the case with the advent of sophisticated spectrum analyzers and associated automatic test equipment. Therefore, the technique of obtaining state space models from frequency response data is of practical interest. Classically, the Inverse Discrete Fourier Transform method (IDFT) is used to transform the frequency response data to time domain data, that is, to transform the frequency response function (FRF) of the system to its pulse response. The pulse response of discrete-time systems is also known as the Markov parameters. The disadvantage of this approach is that the Markov parameter se quence obtained is distorted by time-aliasing effects. ${ }^{17}$ Recently, a method called the State Space Frequency Domain (SSFD) identification algorithm ${ }^{18}$ has been developed. This method can estimate Markov parameters from the FRF without windowing distortion and an arbitrary frequency weighting can be introduced to shape the estimation error. The method uses a rational matrix description (the ratio of a matrix polynomial and a monic scalar polynomial denominator) to curve-fit the frequency data and obtains the Markov parameters from this equation. In obtaining the state space models from the Markov parameters, the Eigensystem Realization Algorithm (ERA), ${ }^{19}$ or its variant ERA/DC, ${ }^{20}$ is used. The disadvantage of this method is that the curve-fitting problem must either be solved by non-linear optimization techniques or by linear approximate algorithms requiring several iterations ${ }^{18}$. Using the same idea as derived for the OKID, a novel method developed in Ref. 21 proposes a simple yet effective way of curve-fitting the FRF data and of constructing the Markov parameters. Instead of using a rational matrix function, this method uses a matrix-fraction for the curve-fitting. Thus the curvefitting is reformulated as a linear problem which can be solved by the ordinary least-squares method in one step; that is, no iteration is required. The method can match the frequency response data perfectly if the FRF is accurate in ideal cases, and will seek an optimal match if noise and/or distortion are involved in the data. This new approach retains all the advantages associated with the SSFD while avoiding the iterative, approximate curve-fitting procedures.

The objective of this paper is to present an overview of the recent advances in system identification for modal testing and control of large structures. We focus on the Observer/Kalman Filter Identification (OKID) $)^{5-11,12,22,23}$, the Observer/Controller Identification (OCID) ${ }^{15,16}$ and the State Space System Identification in the Frequency Domain. ${ }^{18,20}$ Applications to the real aerospace structures will be shown including the Hubble spacecraft telescope ${ }^{14}$ and the active flexible aircraft wing ${ }^{15}$.

\section{OBSERVER/KALMAN FILTER IDENTIFI- CATION}

There are basically two ways to stochastically characterize system uncertainties including process and measurement noises (see Fig. 1). One way is to describe the input and output uncertainties directly in terms of their covariances. Another way is to specify the Kalman filter equation with its steady state Kalman gain, which is a function of the input and output uncertainty covariances. In the OKID, an observer is identified to characterize the input and output uncertainties. If the data length is sufficiently long, and the number of identified observer Markov parameters (pulse response time histories) is sufficiently large, then the identified observer of the system approaches the Kalman filter. 


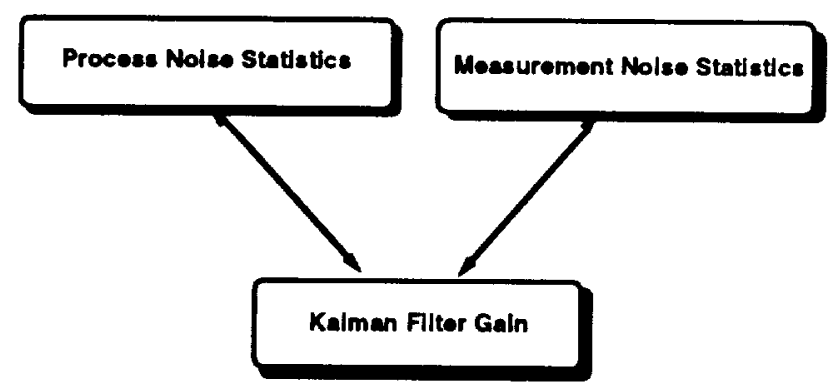

Figure 1: Characterization of system uncertainties

The OKID has two ways of processing the input and output data for system identification. One is the forward-in-time and the other is the backward-intime as shown in Fig. 2. The forward-in-time means

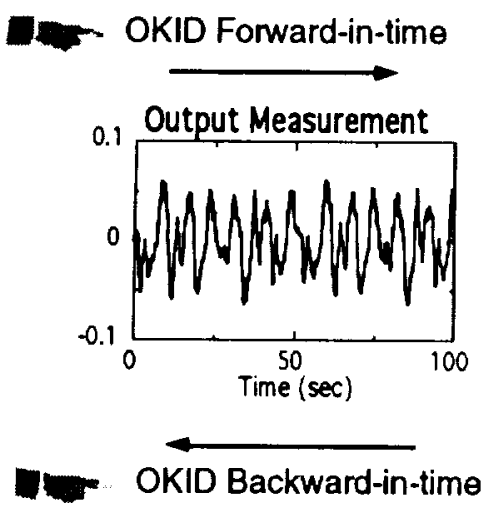

Figure 2: OKID forward and backward

that the current output measurement can be fully estimated by the previous inputs and outputs, and is commonly used for the system identification. If one reverses time in the model to be identified, ${ }^{24,25}$ what were damped true system modes become undamped true system modes, growing as the reversed time increases. Physically, it implies that the current output measurement can be fully estimated by the future inputs and outputs. On the other hand, the noise modes in the forward and backward identification still maintain the property that they are stable. This is intuitively reasonable. If the data set is sufficiently long, an unstable noise mode would predict noise contributions to the pulse response data that grow unbounded as the time step in the data set increases. This is inconsistent with the expected contribution of noise in data. Therefore, the backward identification has the advantage of shifting from positive damping to negative damping of the true system modes to distinguish these modes from noise modes. Real experiences have shown that the backward identification may fail to indicate certain system modes in experimental data, perhaps due to the unmatched uncertainty levels in forward and backward identification.

Given a set of experimental input and output data, the identification algorithm (see Fig. 3) proceeds as follows:

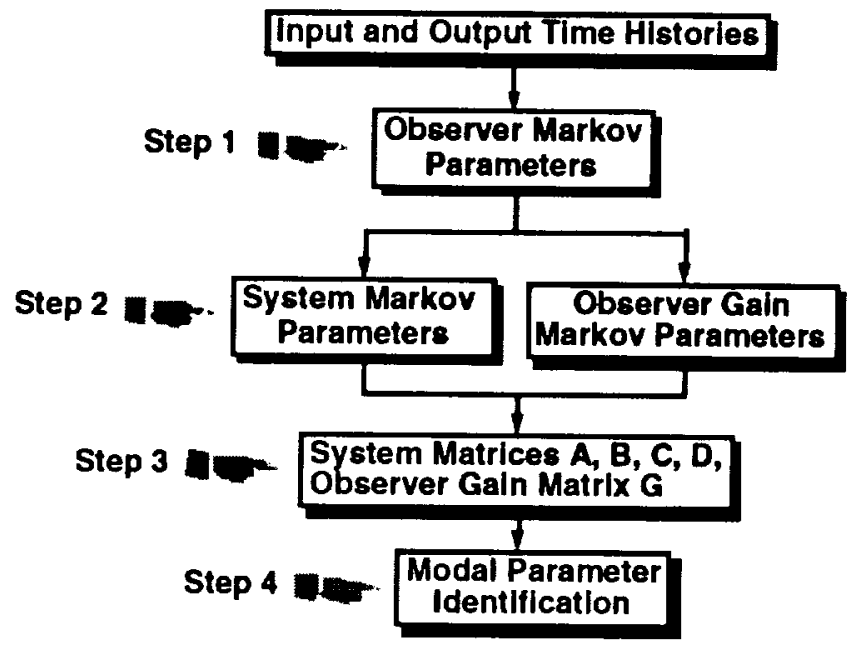

Figure 3: Flow Chart for the OKID

1) Compute observer Markov parameters.

2) Recover the combined system and observer gain pulse response samples from the identified observer Markov parameters.

3) Realize a state space model of the system and the corresponding observer gain from the recovered pulse response samples using ERA or ERA/DC.

4) Find the eigensolution of the realized state matrix and transform the realized model to modal coordinates for modal parameter identification. The modal parameters include frequencies, dampings, and mode shapes at the sensor locations.

To demonstrate the identification procedure using real experimental data, the flight data from the Hubble Space Telescope shown in Fig. 4 is used. There are six gyros located on the Optical Telescope Assembly (OTA) and four torque wheels located on the Spacecraft Subsystem Module (SSM). The OTA is fixed inside the SSM. The gyros are used mainly to measure the motion of the primary mirror. Data from four out of the six gyros are recorded at a time. The measurement resolution is $0.005 \mathrm{arcsec} / \mathrm{sec}$, which implies that the gyro data are not adequate because the requirement is 0.007 arcsec pointing. The angular rates, which are measured along the four gyro directions, are combined and transformed using least-squares to recover the three rates in vehicle coordinates. Leastsquares is used to smooth the poor resolution of the data. The input commands are given in terms of angular acceleration in the three rotational vehicle coordinates and then projected on the four torque wheel 


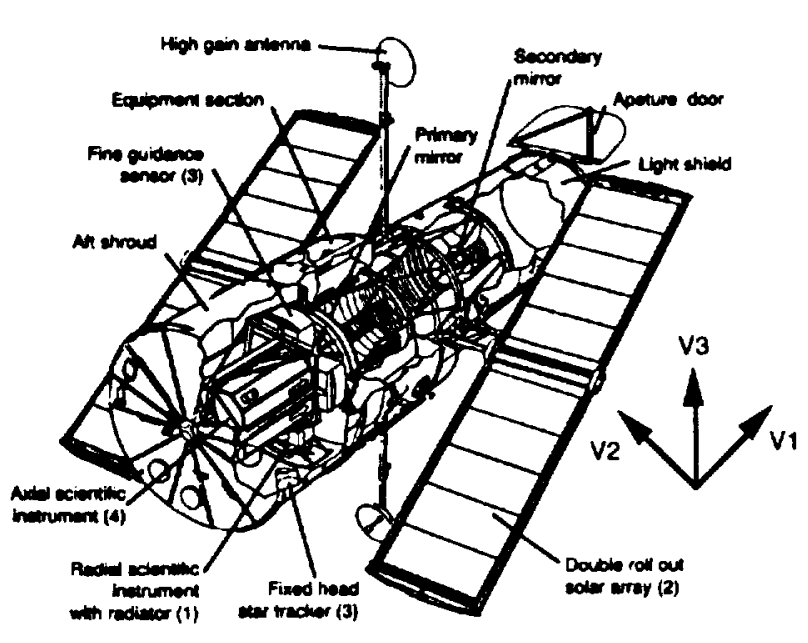

Figure 4: Hubble Spacecraft Telescope

axes to excite the telescope mirror and the spacecraft. The data were sampled at $40 \mathrm{~Hz}$. Pulses combined with sine-sweeping in the middle of an excitation period $(50.975 \mathrm{sec})$ were used as input commands to the torque wheels. The excitation period was repeated six times for a total of approximately 12,000 samples taken for each experiment. The experiment was repeated three times for the other two vehicle coordinates. As a result, there were three inputs and four outputs for a total of three sets of 12,000 input samples and twelve sets of 12,000 output samples to be used for identification of vibration parameters.

The usual practice of modal parameter identification uses the Fast Fourier Transforms (FFT) of the inputs and measured outputs to compute the pulse response sequence (system Markov parameters). In contrast, the OKID uses an asymptotically stable observer to form a stable state space discrete model for the system to be identified. The primary purpose of introducing an observer is to compress the data and improve system identification results in practice.

The first step is to compute the observer Markov parameters. As shown in Fig. 5, the input and output time histories are several orders longer than the observer pulse response sequence (observer Markov parameters). For illustration, only the input and output time histories from the first vehicle axis are shown. The modal parameters which are excitable by the inputs and measurable by the output sensors are embedded in the identified observer Markov parameters.

The second step is to compute system pulse responses (system Markov parameters) and observer gain pulse responses (observer gain Markov parameters). From the identified observer Markov parameters, the system Markov parameters and the observer gain Markov parameters can be easily computed. The results for the first vehicle axis, V1, are graphically

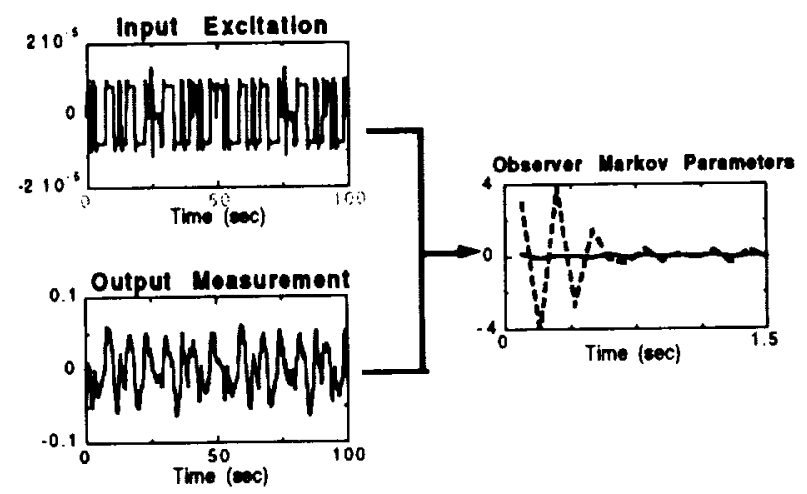

Figure 5: Computation of observer Markov parameters

shown in Fig. 6. Although the number of identified ob-

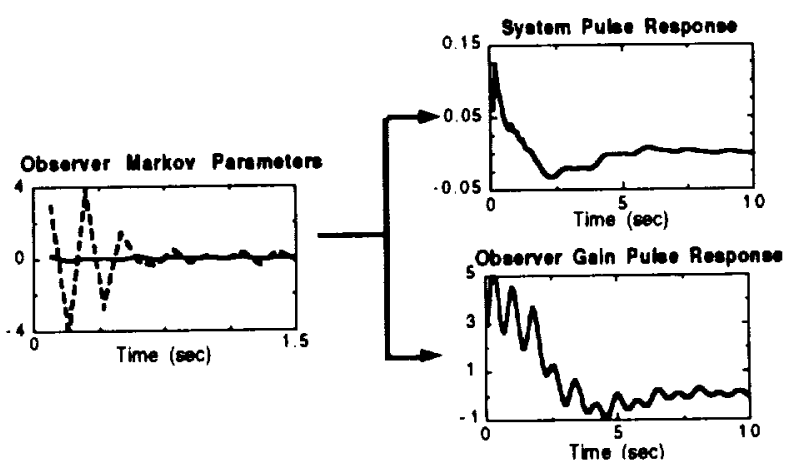

Figure 6: Computation of system and observer gain Markov parameters

server Markov parameters is finite and generally very small, the number of system Markov parameters can be as large as desired. Note that the maximum number of independent system Markov parameters is equal to the number of identified observer Markov parameters. To solve for more system Markov parameters than the number of identified observer Markov parameters, simply set the extra observer Markov parameters to zero.

The third step as shown in Fig. 7 is to compute the system matrices and the observer gain matrix. Knowledge of the actual system Markov parameters and the observer gain Markov parameters allows one to use the ERA or ERA/DC to obtain a state space realization of the system of interest. Modal parameters including natural frequencies, damping ratios, and mode shapes can then be found from the system matrices. The identified observer gain is related to the steady state Kalman filter gain which may be used to characterize the system uncertainties and measurement noises.

The system order identified from ERA/DC, after some singular values truncation, was chosen to be 30 for the realization of system matrices. Seven dominant modes were identified as shown in Table 1. The Mode $\mathrm{SV}$ in the table describes the singular value contribu- 


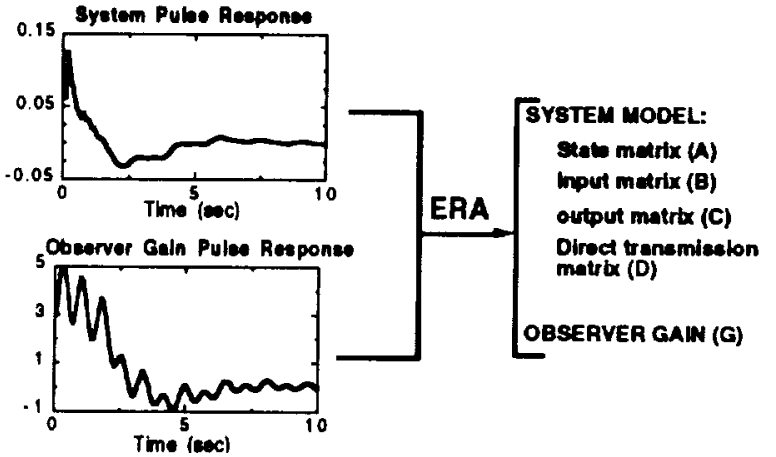

Figure 7: Computation of system and observer gain matrices

Table 1: Identified modal parameters for the Hubble Space Telescope

\begin{tabular}{||c|c|c|c||}
\hline $\begin{array}{c}\text { Mode } \\
\text { No. }\end{array}$ & $\begin{array}{c}\text { Frequency } \\
(\mathrm{Hz})\end{array}$ & $\begin{array}{c}\text { Damping } \\
(\%)\end{array}$ & $\begin{array}{c}\text { Mode } \\
\text { SV }\end{array}$ \\
\hline 1 & 0.147 & 55.6 & 0.76 \\
2 & 0.155 & 58.4 & 0.98 \\
3 & 0.169 & 67.4 & 1.00 \\
4 & 0.633 & 57.3 & 0.68 \\
5 & 1.273 & 4.06 & 0.37 \\
6 & 2.433 & 5.23 & 0.02 \\
7 & 2.822 & 6.33 & 0.01 \\
\hline \hline
\end{tabular}

tion of each individual mode to the system Markov parameters. It has been normalized relative to the maximum singular value. The first three modes are attitude modes. The $0.633 \mathrm{~Hz}$ mode is believed to be an in-plane bending mode of the solar array, the $1.273 \mathrm{~Hz}$ mode is a coupled solar and membrane mode, and the $2.433 \mathrm{~Hz}$ mode is the first mode of the primary deployment mechanism with the solar array housing attached. The identified dampings are higher than expected because there is an attitude control for maneuvering during testing, as well as inherent mechanical friction of the solar array mechanism.

The left figure in Fig. 8 shows the excitation input signal including pulse combined with sine-sweeping components in the middle of an excitation period for the first vehicle axis. The right figures in Fig. 8 show overlapping 50 seconds of the reconstruction from the identified system models, and the test data for the first vehicle axis. The upper right corner in Fig. 8 shows the predicted output in comparison with the real output data. The lower right corner in Fig. 8 shows the estimated output in comparison with the real output data. The predicted output is the output reconstructed from the identified model only whereas the estimated out-

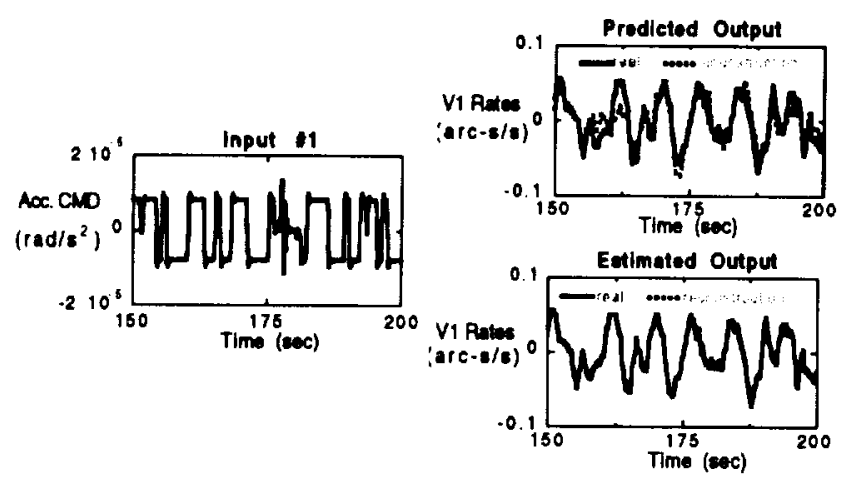

Figure 8: Comparison of predicted and estimated output

put is the output reconstructed from the identified observer. There are visible differences in the predicted and estimated outputs. Comparison of the observer output with the measured response shows extremely good agreement, indicating that the observer is correcting for the system uncertainties including nonlinearities. The covariance of the estimated output residuals is about three orders less than the predicted output residuals. Similar results of the predicted and estimated outputs were obtained for the second and third vehicle axes, and thus are not shown in this example.

Figure 9 shows the comparison of the forward and backward identification results. The left figure shows
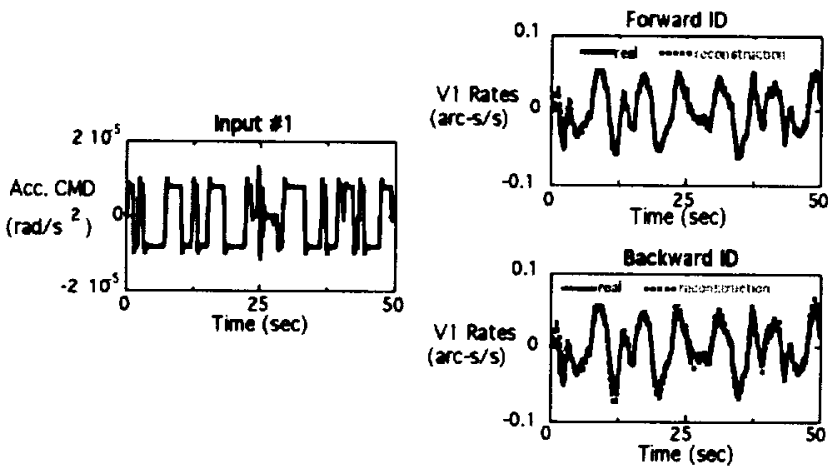

Figure 9: Comparison of OKID-forward and OKIDbackward results

the excitation input signal including pulse combined with sine-sweeping components in the middle of an excitation period (50.975 sec). The figures on the right hand side show overlapping 50 seconds of the reconstruction from the identified forward and backward system models, and the test data for the first vehicle axis. There are some visible differences in the backward identification between test and reconstruction but overall the map from the input to the output is reasonably well. The forward identification is somewhat better than the backward identification in damp- 
ing estimation. The damping ratio estimated from the backward approach appears to be a little low. It is important that the system model be accurate because it is this part that is used as a model for control design.

\section{OBSERVER/CONTROLLER IDENTIFICA- TION}

This section presents a technique that identifies a control system operating under closed-loop conditions with an existing feedback controller, which may or may not include feedback dynamics. The controller and the open-loop system dynamics are assumed to be unknown. The closed-loop system is excited by a known excitation signal, and the closed-loop system output responses and the feedback signal are measured. A schematic diagram of the existing or actual closed-loop system is given in Fig. 10 which shows the measured

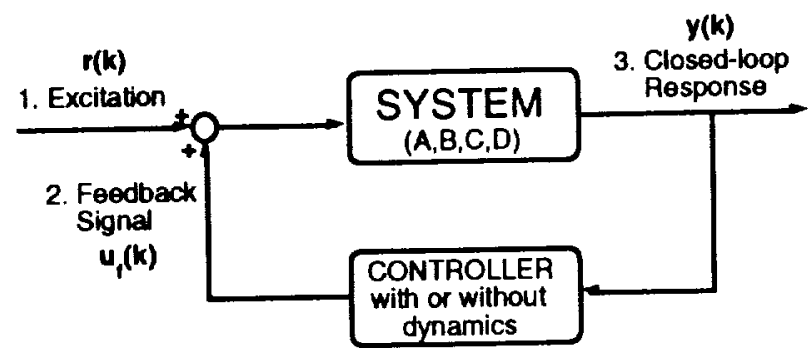

Figure 10: Existing (Actual) control system

quantities, and the open-loop system in state-space representation given by the matrices $A, B, C, D$. An algorithm is developed to identify the open-loop system, an observer gain, and the existing controller gain matrices from closed-loop test data which include the time histories of the excitation signal, the resulting closed-loop response, and the feedback control signal. The technique assumes the identified controller to be of a full state feedback type. A schernatic diagram of the identified or effective closed-loop system is shown in Fig. 11, where $A, B, C, D$ again represent the identified

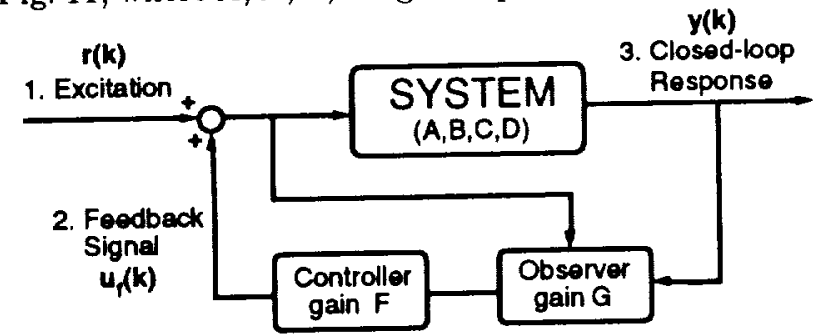

- Find $A, B, C, D, G$, and $F$ from data at points 1,2 , and 3

Figure 11: Identified (Effective) control system

open-loop system; $G$ and $F$ represent the identified observer and controller gains, respectively. The method first identifies the Markov parameters of a closed-loop observer, which in turn produce the Markov param- eters for an observer, the open-loop system, and the controller.

The OCID here means the Observer/Controller Identification. Given a set of excitation signal, feedback signal, and closed-loop response data, the identification algorithm (see Fig. 12) proceeds as follows.

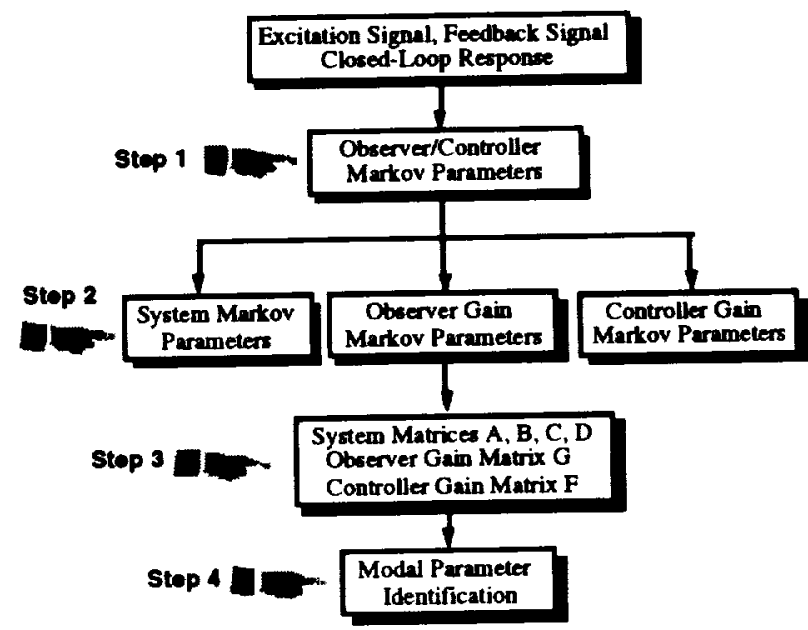

Figure 12: Flow chart for the OCID

1) Compute observer/controller Markov parameters.

2) Recover system, observer gain, and controller Markov parameters

3) Realize a state space model of the system, the corresponding observer and controller gains from the recovered sequence of the system and observer gain Markov parameters by using ERA or ERA/DC.

4) Find the eigensolution of the realized state matrix and transform the realized model to modal coordinates for modal parameter identification. The modal parameters include frequencies, dampings, and mode shapes at the sensor locations.

The OCID method is illustrated by using actual aircraft flutter test data. ${ }^{26}$ Experimental data was obtained from wind tunnel tests of an aeroelastic model with active flutter control operating (see Fig. 13). The model, known as the Active Flexible Wing (AFW), has a digital controller which suppresses flutter by properly phased commands to actuators of eight control surfaces on the wing leading and trailing edge surfaces. During flutter suppression control law testing, acceleration signals from sensors distributed on the model were first filtered for anti-aliasing and then quantized 


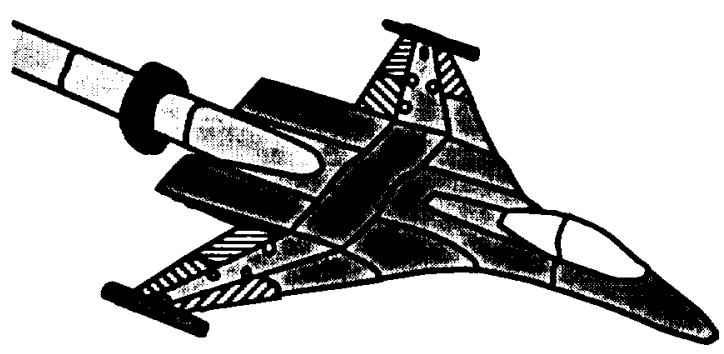

Figure 13: Active flexible wing

at a $200 \mathrm{~Hz}$ sample rate. The quantized signals obtained from both sides of the model were then symmetrized in pairs. These symmetrized signals became the inputs to the symmetric and antisymmetric flutter suppression control laws and also the source of the closed-loop response time histories to be used for the identification process. Output signals of the feedback control laws and independent input excitation to the wing provided the remaining time histories necessary for identification of the closed-loop control system. During tests, each of the actuator inputs was excited individually by adding the excitation signal to the feedback control output signal. This procedure allowed the generation of all the responses necessary to identify the multi-input/output control system. The excitation signals themselves were either logarithmic sine sweeps or so-called pseudo-random noise. The excitation signal, the resultant closed-loop response time histories, and the feedback control signal were used with the OCID technique to identify all of the elements of the AFW model including the open-loop system matrices, an observer gain, and the existing controller gains. The flutter mode is then identified by solving the eigenvalues of the open-loop state matrix.

Seven sets of experimental data were used corresponding to different dynamic pressure conditions, 175 pounds per square foot (psf), $200 \mathrm{psf}, 230 \mathrm{psf}, 240 \mathrm{psf}$, $250 \mathrm{psf}, 260 \mathrm{psf}$ and $280 \mathrm{psf}$ respectively. Results for the $260 \mathrm{psf}$ condition are shown in the following unless other wise specified. The number of data points used in this case is 600 , with a sampling interval of $0.005 \mathrm{sec}$. apart $(200 \mathrm{~Hz}$ sampling rate). The actual time histories used in the identification and the identification results are shown in Fig. 14, which are discussed in more details below.

From the data histories shown in Fig. 14 for the first 2 seconds, 30 observer/controller Markov parameters are computed. The identified observer/controller Markov parameters are shown on the middle of Fig. 14 for a duration of $0.15 \mathrm{sec}$. There are four curves in this plot. Using the identified observer/controller Markov parameters, the system, observer gain, controller gain,

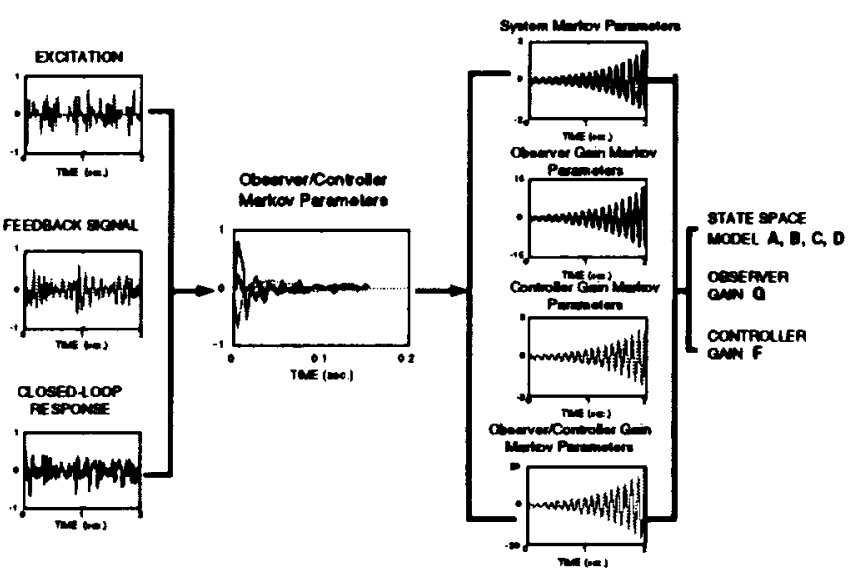

Figure 14: Identified Markov parameters

and observer/controller gain Markov parameters are computed, which are shown on the right hand side of Fig. 14. Note that these time histories are not limited to the $0.15 \mathrm{sec}$. duration. In fact, the system, observer gain, controller gain, and observer/controller gain Markov parameters can be computed for any duration as desired. The pulse responses increase in amplitudes with time, revealing open-loop instability.

Using the computed Markov parameters, a state space model relating the system, the controller gain, and the observer gain are then computed. The system Markov parameters are simply its pulse response samples. The flutter mode is then identified by solving the eigenvalues of the open-loop state matrix.

Figure 15 shows that the identified flutter mode

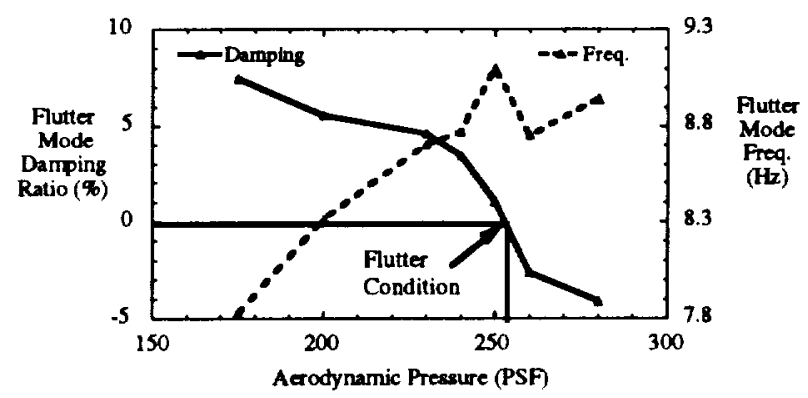

Figure 15: Identified Markov parameters

for the 260 psf condition has an open-loop frequency of $8.78 \mathrm{~Hz}$ and $3.34 \%$ negative damping, implying open-loop instability. This example illustrates the case where open-loop identification may not be possible or practical for such a system. Similar analysis performed on the six remaing sets of data revealed that the identified flutter mode for the $250 \mathrm{psf}$ condition has an open-loop frequency of $9.06 \mathrm{~Hz}$ and $0.26 \%$ negative damping, indicating marginal open-loop instabil- 
ity. The final $280 \mathrm{psf}$ condition was identified to have an open-loop frequency of $8.76 \mathrm{~Hz}$ and $5.73 \%$ negative damping, indicating even greater open-loop instability. Comparison of the identified with the analytical results showed excellent agreement in frequencies and damping, indicating a coalescing mode switch in frequency.

In general, a specific (or existing) observer is not identifiable because the observer becomes ineffective when the transient responses decay out and the errors between the true states and the estimated states become dominated by the system uncertainties and measurement noises. Therefore, from given excitation signals, feedback signals, and measurement data, one identifies an effective observer determined by the system uncertainties and measurement noises, instead of the specific observer. However, this does not influence the identification of the open-loop system and the feedback controller gain. When the data length is sufficiently long, and the number $p$ is chosen to be sufficiently large, then the identified observer tends to a Kalman filter which may not be the observer given by the controller designer. Also, numerical studies indicate that this technique particularly works well for unstable systems because the signal to noise ratio for an unstable mode is generally higher than that for a stable mode.

\section{FREQUENCY-DOMAIN SYSTEM IDENTI- FICATION}

The objective of frequency domain state space system identification is to identify state space models from the given frequency response data - the frequency response functions (FRF). The transfer function of a multi-input and multi-output linear system has left and right matrix-fraction descriptions. From the left matrix-fraction description (LMFD), one can derive a simple observable canonical form, whereas from the right matrix-fraction description (RMFD) one can derive a simple controllable canonical form, which is shown as follows. Neither observable nor controllable canonical form is a minimum realization. The minimum realization means a model with the smallest state space dimensions among all realizable systems that have the same input-output relations. Instead of computing canonical-form realizations, one may compute the system Markov parameters and then obtain a minimum state space realization using ERA.

The computational steps for the matrix-fraction description methods are shown in Fig. 16 and summarized as follows:

1. Determine frequency response functions.

2. Curve-fit the frequency response function using the left or right matrix-fraction description method.

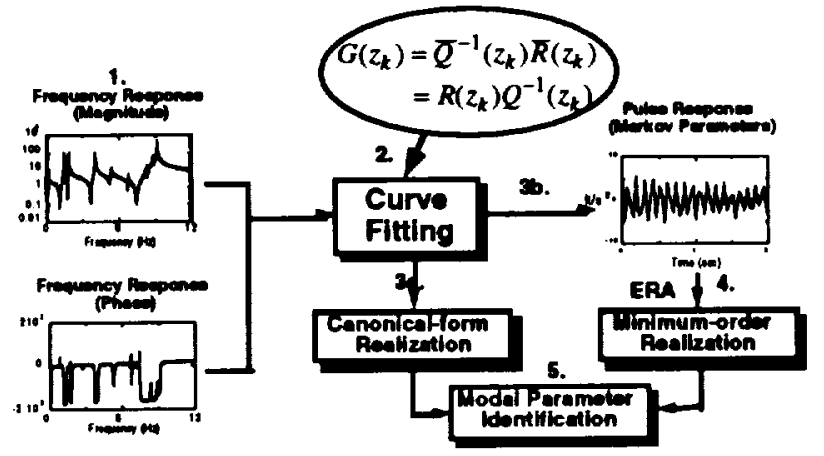

Figure 16: Computational Steps for left of right matrix-fraction description method.

3a. Construct a canonical-form realization. If the left matrix-fraction description method is used, an observable canonical form will be obtained. If the right matrix-fraction description method is used, a controllable canonical form will be obtained.

3b. Compute system Markov parameters as many as desired if a model with minimum order is to be determined.

4. Determine a minimum order realization from the computed system Markov parameters by using a minimum realization technique such as ERA.

5. Find the eigensolution of the realized state matrix and transform the realized model to modal coordinates for modal parameter identification. The modal parameters include frequencies, dampings, and mode shapes at the sensor locations.

The left matrix-fraction description method is illustrated by using the structure shown in Fig. 17 which is a NASA testbed ${ }^{27}$ to study the controls and structures interaction problem. The system has eight inputs and

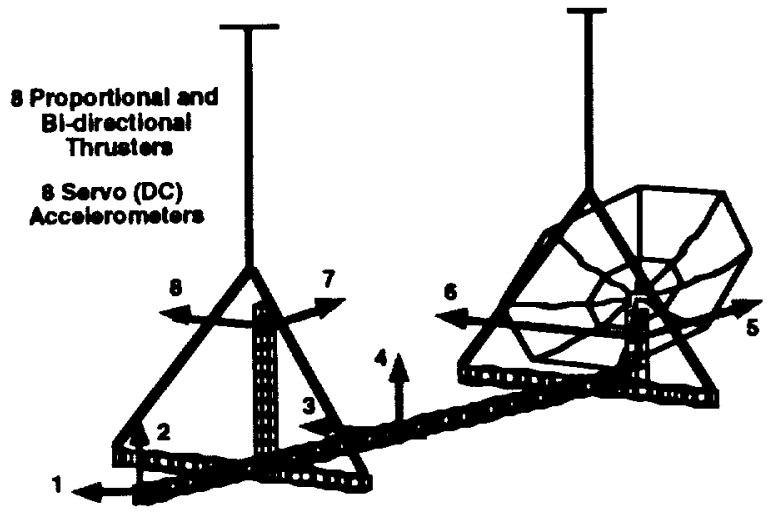

Figure 17: A NASA large space structure testbed.

eight collocated outputs for control. The inputs are 
air thrusters and the outputs are accelerometers. The locations of the input-output pairs are depicted in Fig. 17. In this example, the structure was excited using random input signals to four thrusters located at positions $1,2,6,7$. The input and output signals were filtered using low-pass digital filters with the range set to $78 \%$ of the Nyquist frequency $(12.8 \mathrm{~Hz})$ to concentrate the energy in the low frequency range below $10 \mathrm{~Hz}$. A total of 2048 data points at a sampling rate of $25.6 \mathrm{~Hz}$ from each sensor are used for identification.

Sixteen FRF's from four input and output pairs located at positions 1, 2, 6, 7 are simultaneously used to identify a state space system model to represent the testbed. The order of the matrix polynomial is set to 25 , which is sufficient to match as many as $\mathbf{5 0 \text { modes }}$ (a system of dimension 100). A state space model is obtained using ERA/DC with the system order assigned to 100 . The reconstructed frequency response data (dash lines) are compared with the experimental data (solid lines) in Figs. 18 and 19.
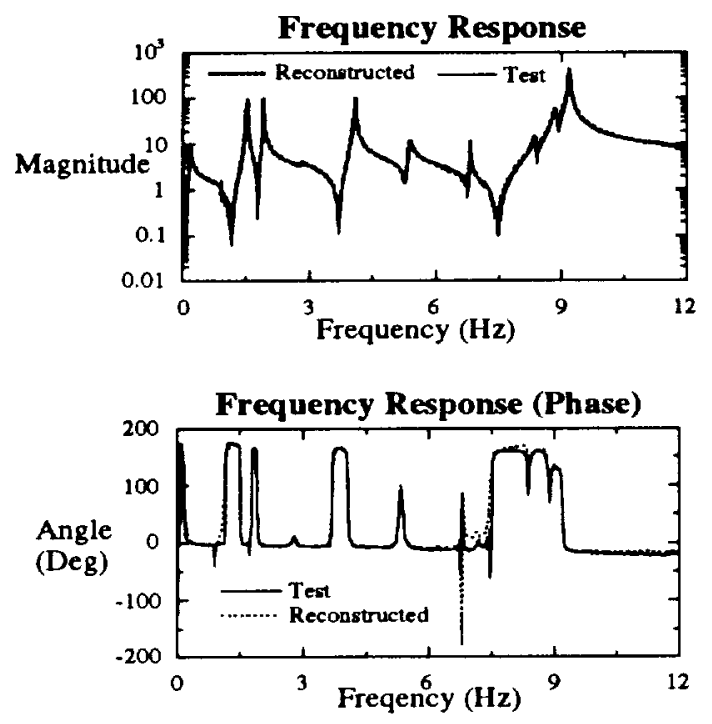

Figure 18: Comparison of the test (solid line) and reconstructed (dash line) input-1/output-1 FRF's. The reconstructed FRF is obtained using the identified system matrices.

Figure 18 is the frequency response of output 1 with respect to input 1 , representing a case of a strong signal, while Fig. 19 is the frequency response of output 2 with respect to input 1 , representing a case of a weak signal. The signal is weak because sensor 2 is orthogonal to input 1 . Similar results are obtained for other input/output pairs which are not shown. The results show that the matching is better for the strong signal cases. This is expected because the strong signal has a larger signal-to-noise ratio than the weaker signal. The results for other input-output pairs are
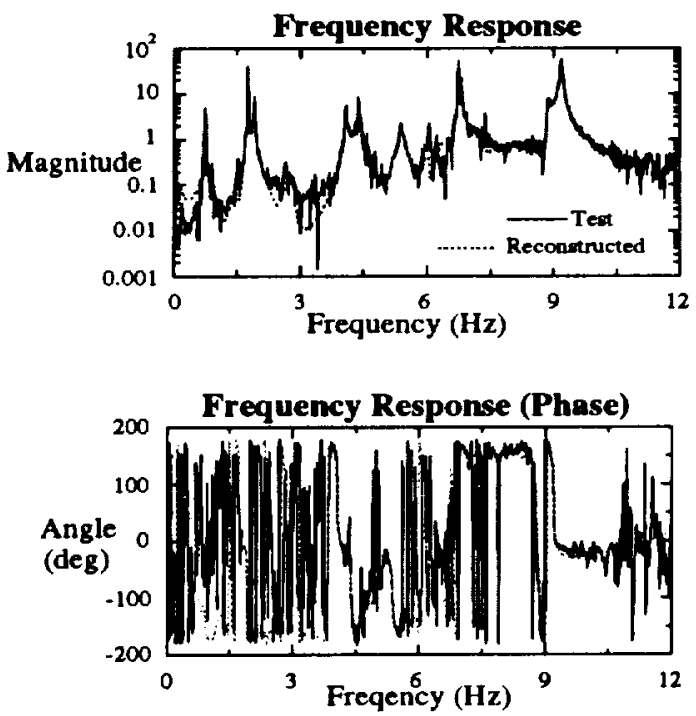

Figure 19: Comparison of the test (solid line) and reconstructed (dash line) input-1/output-2 FRF's. The reconstructed FRF is obtained using the identified system matrices.

similar and hence omitted.

\section{CONCLUDING REMARKS}

The field of system identification has expanded continuously and extensively over two decades. This growth is largely associated with corresponding improvements in computer capabilities. These increases in computer capability have permitted more accurate and complete testing and data analysis to occur. Algorithms and approaches thought too extensive in the past are now feasible. In particular, many important numerical tools have been developed including the singular value decomposition which is an essential tool in the derivation of system identification methods. In this paper, an overview of several recently developed techniques are presented including the Observer/Kalman Filter Identification, the Observer/Controller Identification, and the State-Space System Identification in the Frequency Domain. These techniques have been successfully applied to many aerospace structures. However, complex, built-up structures still pose a significant challenge to the best ground-based methodology now available.

\section{REFERENCES}

1. Ljung, L., System Identification: Theory for the User, Prentice-Hall, Inc., Englewood Cliffs, New Jersey 07632.

2. Juang, J.-N., and Pappa, R. S., "A Comparative Overview of Modal Testing and System Identifi- 
cation for Control of Structures," Shock and Vibration Digest, Vol. 20, No. 5, May 1988, pp. 4-15.

3. Pappa, R. S., and Juang, J.-N., "Some Experiences with the Eigensystem Realization Algorithm," Sound and Vibration, Vol. 22, No. 1, Jan. 1988, pp. 30-35.

4. Juang, J. N., Horta, G. H., and Longman, R. W., "Input/Output System Identification: Learning From Repeated Experiments," Mechanics and Control of Large Space Structures edited by John L. Junkins, AIAA Monograph on Progress in Astronautics and Aeronautics, Vol 29, 1990, pp. 87-99.

5. Chen, C.-W., Huang, J.-K., Phan, M., and Juang, J.-N., "Integrated System Identification and Modal State Estimation for Control of Large Flexible Space Structures," Journal of Guidance, Control, and Dynamics, Vol. 15, No. 1, pp. 8895, Jan.-Feb. 1992.

6. Phan, M., Juang, J.-N., and Longman, R.W., "Identification of Linear Multivariable Systems from a Single Set of Data by Identification of Observers with Assigned Real Eigenvalues," Journal of Astronautical Sciences, Vol. 40, No. 2, April-June 1992, pp. 261-279.

7. Phan, M., Horta, L. G., Juang, J.-N., and Longman, R.W., "Linear System Identification Via an Asymptotically Stable Observer," NASA Technical Paper 3164, June 1992.

8. Juang, J.-N., Phan, M., Horta, L. G., and Longman, R.W.., "Identification of Observer/Kalman Filter Markov Parameters: Theory and Experiments," Proceedings of the AIAA Guidance, Navigation and Control Conference, New Orleans, Louisiana, Aug. 1991, and also to appear in Journal of Guidance, Control and Dynamics.

9. Phan, M., Horta, L. G., Juang, J.-N., and Longman, R. W., "Improvement of Observer/Kalman Filter Identification by Residual Whitening," Proceedings of Eighth VPIE SU Symposium edited by L. Meirovitch, Blacksburg, Virginia, May 6-8, 1991, pp. 467-478.

10. Chen, C.-W., Huang, J.-K., and Juang, J.N., "Identification of Linear Stochastic System through Projection Filters," Proceedings of the AIAA 33nd Structures, Structural Dynamics \& Materials Conference, Dallas, TX, April 13-15, 1992, pp. 2320-2340.

11. Chen, C. W., Lee, G., and Juang, J.-N., "Several Recursive Techniques for Observer/Kalman Filter System Identification From Data," Paper No. 92-4386, AIAA Guidance, Navigation and Con- trol Conference, Hilton Head, South Carolina, Aug. 10-12, 1992

12. Juang, J.-N. and Phan, M., "Linear System Identification via Backward Observer Models," NASA Technical Memorandum TM-107632, May 1992.

13. Scott, M., Gilbert, M., and Demeo, M., "Active Vibration Damping of the Space Shuttle Remote Manipulator System," Proceedings of the AIAA Guidance, Navigation and Control Conference, New Orleans, Louisiana, Aug. 1991.

14. Juang, J.-N., Horta, L. G., Belvin, W. K., Sharkey, J., and Bauer, F. H., "An Application of the Observer/Kalman Filter Identification (OKID) Technique to Hubble Flight Data," NASA/DoD Controls-Structures Interaction (CSI) Technology Conference, March 35, 1992, Harvey's Resort Hotel, Lake Tahoe, Nevada.

15. Juang, J.-N., and Phan, M., "Identification of system, "Observer, and Controller from ClosedLoop Experimental Data," Paper No. 92-4620, AIAA Guidance, Navigation and Control Conference, Hilton Head, South Carolina, Aug. 10$12,1992$.

16. Phan, M., Juang, J.-N., Horta, L. G., Longman, R. W., "System Identification from Closed-Loop Data with Known Output Feedback Dynamics," NASA Technical Memorandum 107604, March 1992.

17. Oppenheim, A. V. and Schafer, R. W., Digital Signal Processing, Prentice-Hall Inc. Englewood Cliffs, New Jersey., 1975.

18. Bayard, D. S., "An Algorithm for State Space Frequency Domain Identification without Windowing Distortions," Proceedings of the Control and Decision Conference, December 1992.

19. Juang, J.-N., and Pappa, R.S., "An Eigensystem Realization Algorithm for Modal Parameter Identification and Model Reduction," Journal of Guidance, Control, and Dynamics, Vol. 8, No. 5, Sept.-Oct. 1985, pp. 620-627.

20. Juang, J.-N., Cooper, J.E., and Wright, J.R., "An Eigensystem Realization Algorithm Using Data Correlations (ERA/DC) for Modal Parameter Identification," Control-Theory and Advanced Technology, Vol. 4, No. 1, 1988, pp. 5-14.

21. Chen, C. W., Juang, J.-N., and Lee, G., "Frequency Domain State-Space System Identification," NASA Technical Memorandum 107659, June 1992.

22. Phan, M., Horta, L.G., Juang, J.-N., and Longman, R.W., "Improvement of Observer/Kalman 
Filter Identification by Residual Whitening," AIAA Guidance, Navigation and Control Conference, Hilton Head, South Carolina, Aug. 1992.

23. Juang, J.-N., Horta, L.G., and Phan, M., "User's Guide for System/Observer/Controller Identification Toolbox," NASA Technical Memorandum 107566, 1992.

24. Hollkamp, J.J. and Batill, S.M., "Automated Parameter Identification and Order Reduction for Discrete Series Models," AIAA Journal, Vol. 29, No. 1, 1991.
25. Hollkamp, J.J. and Batill, S.M., "Structural Identification Using Order Overspecified TimeSeries Models," to appear in Journal of Dynamics Systems, Measurement, and Control, 1992.

26. Perry III, B., Cole, S. R., and Miller, G. D., "A Summary of the Active Flexible Wing Program," AIAA Paper No. 92-2080, AIAA Dynamics $S p e-$ cialists Conference, Dallas, Texas, April 1992.

27. Belvin, W. K., Elliott, K. B., Horta, L. G., Bailey, J. P., Bruner, A. M., Sulla, J. L., Won, J., Ugoletti, R. M. "Langley's CSI Evolutionary Model: Phase 0," NASA Technical Memorandum 104165, November 1991. 



\section{INTEGRATED CONTROLS-STRUCTURES DESIGN}


